Man and Nature

MAN AND NATURE

L'homme et la nature

L'HOMME ET LA NATURE

\title{
La Rhétorique amoureuse dans Les Égarements du coeur et de l'esprit
}

\section{Jean Terrasse}

Volume 1, 1982

URI : https://id.erudit.org/iderudit/1011788ar

DOI : https://doi.org/10.7202/1011788ar

Aller au sommaire du numéro

\section{Éditeur(s)}

Canadian Society for Eighteenth-Century Studies / Société canadienne d'étude du dix-huitième siècle

\section{ISSN}

0824-3298 (imprimé)

1927-8810 (numérique)

Découvrir la revue

\section{Citer cet article}

Terrasse, J. (1982). La Rhétorique amoureuse dans Les Égarements du coeur et de l'esprit. Man and Nature / L'homme et la nature, 1, 21-29.

https://doi.org/10.7202/1011788ar

Copyright @ Canadian Society for Eighteenth-Century Studies / Société canadienne d'étude du dix-huitième siècle, 1982
Ce document est protégé par la loi sur le droit d'auteur. L'utilisation des services d'Érudit (y compris la reproduction) est assujettie à sa politique d'utilisation que vous pouvez consulter en ligne.

https://apropos.erudit.org/fr/usagers/politique-dutilisation/ 


\section{La Rhétorique amoureuse dans Les Égarements du coeur et de l'esprit}

L'art d'aimer, dans Les Egarements du coeur et de l'esprit, se présente comme une rhétorique.

Que le roman, à l'époque de Crébillon, reste tributaire d'une définition rhétorique de la littérature, est un fait bien établi. Nous savons, grâce à Georges May, que l'évolution du genre s'explique alors en grande partie par la nécessité où se trouvent les romanciers "de justifier leurs ouvrages soit du point de vue esthétique, soit du point de vue moral," Les Egarements paraissent au moment où la querelle du roman, mettant aux prises Lenglet du Fresnoy, le P. Bougeant (qui attaque Crébillon), le P. Porée, atteint son apogée: à partir de 1737, "la publication des romans," selon Servais Etienne, sera "soumise en France à un régime d'exception qui équivaut presque à l'interdiction pure et simple."

Les attaques contre le roman dans la première moitié du XVIII ${ }^{\mathrm{e}}$ siècle fixent l'attention sur les deux fonctions traditionnellement assignées à la littérature: plaire et instruire. Ces objectifs obligent à considérer la littérature comme un discours persuasif et à insister sur le vraisemblable. En réalité, ils imposent à l'écrivain des impératifs contradictoires. Le désir de persuader le fait recourir à des procédés rhétoriques, mais le souci de la vraisemblance le force à en rejeter l'usage abusif, à la limite, à les dénoncer. Todorov a montré qu'une des tendances de la rhétorique a été d'opposer le discours figuré à un discours naturel impossible à décrire; tendance qui se fait jour, entre autres, dans le célèbre traité Des Tropes de Du Marsais. ${ }^{3} \mathrm{~A}$ la fois descriptive et normative, la rhétorique repose sur une dialectique du naturel et de l'artificiel. La théorie de l'art-imitation joue sur la distinction entre vérité et vraisemblance. Si la littérature se situe du côté du vraisemblable, c'est pour valoriser, en creux, le champ de la vérité, pour suggérer que rien ne peut, au fond, remplacer la nature: Derrida a parlé, pour caractériser cette littérature, de "métaphysique de la présence."

Plaire et instruire sont les deux buts avoués par Crébillon dans la Préface de son roman. "L'homme qui écrit," précise-t-il, "ne peut avoir que deux objets: l'utile et l'amusant." Et l'application de ces deux critères au roman l'amène à écarter les ouvrages qui ne satisfont pas aux normes de la crédibilité:

Le Roman, si méprisé des personnes sensées, et souvent avec justice, serait peut-être celui de tous les genres qu'on pourrait rendre le plus utile, s'il était bien manié, si, au lieu de le remplir de situations ténébreuses et 
forcées, de Héros dont les caractères et les aventures sont toujours hors du vraisemblable, on le rendait, comme la comédie, le tableau de la vie humaine, et qu'on y censurât les vices et les ridicules. ${ }^{5}$

On pourrait songer à dresser la liste des figures les plus fréquemment employées dans Les Egarements du coeur et de l'esprit; elle montrerait ce que Crébillon doit à l'esthétique de son temps. Comme dans La Princesse de Clèves, les caractérisations physiques - d'ailleurs fort rares - se font par le moyen d'hyperboles. Après la soirée passée à l'Opéra, Meilcour se rend chez Mme de Lursay, qui pour l'attendre, a revêtu "le déshabillé le plus noble et le plus galant" (p. 107). Dans un entretien avec sa mère, il résume en ces termes l'impression produite par la beauté de Mlle de Théville; et Crébillon ajoute à l'hyperbole la gradation et la répétition: "Sa figure, son maintien, son esprit, tout plait en elle, tout y attache. Ce sont les plus beaux yeux! Les plus tendres! Les plus touchants!" (p. 179).

On ne s'étonnera pas de trouver dans Les Egarements un grand nombre de maximes. Expliquant, tout au début du roman, pourquoi il a choisi de séduire Mme de Lursay, Meilcour remarque: "On s'attache souvent moins à la femme qui touche le plus, qu'à celle qu'on croit le plus facilement toucher" (p. 49). Plus loin: "Les leçons et les exemples sont peu de chose pour un jeune homme" (pp. 51-52). Souvent, les maximes s'insèrent dans le mouvement naturel de la narration, dont elles soulignent l'exemplarité:

Je passe sur les sentiments qui m'occupèrent cette nuit-là. Il n'y a pas d'homme sur la terre assez malheureux pour n'avoir jamais aimé, et aucun qui ne soit par conséquent en état de se les peindre. Si la vanité seule avait pu satisfaire mon coeur, il aurait sans doute été moins agité. (p. 177)

Emaillant dialogues et portraits, les maximes forment la vraie trame du roman et contribuent fortement à sa cohérence. Aristote les définit comme des enthymèmes abrégés; il est donc normal qu'elles jouent un rôle important dans l'argumentation. Vu sous un certain angle, le roman de Crébillon aboutit même à la production de maximes: le discours de Versac à Meilcour, dans la troisième partie, se compose de préceptes, règles, conseils, à l'usage du néophyte soucieux d'apprendre à se comporter dans le monde.

Prise dans le contexte narratif, qui l'enferme dans les limites de l'espace et $\mathrm{du}$ temps, l'argumentation rhétorique donne naissance au portrait. Le portrait est-il autre chose qu'une maxime appliquée au caractère des personnages et aux situations où ils évoluent? Pour reprendre une formule percutante d'André Breton, le romancier n'a "qu'à mettre l'aiguille de "Beau fixe" sur "Action" et le tour sera joué." $\mathrm{Si}$ le portrait relativise la pensée de l'écrivain - dans la mesure où le romancier est un philosophe qui n'a pas réussi à "décoller" du quotidien - on peut penser aussi qu'il généralise les particularités du personnage et les expériences des différents moments de sa vie. Voyons le portrait de Mme de Senanges:

Madame de Senanges avait été jolie, mais ses traits étaient effacés. Ses yeux languissants et abattus n'avaient plus ni feu ni brillant. Le fard qui achevait de flétrir les tristes restes de sa beauté, sa parure outrée, son 
maintien immodeste, ne la rendaient que moins supportable. C'était une femme enfin à qui, de toutes ses anciennes grâces, il ne restait plus que cette indécence que la jeunesse et les agréments font pardonner, quoiqu'elle déshonore l'un et l'autre, mais qui, dans un âge avancé, ne présente plus aux yeux qu'un tableau de corruption qu'on ne peut regarder sans horreur (pp. 151-152).

Ce portrait, l'un des plus "concrets" du roman, permet cependant de prévoir l'essentiel des actions de Mme de Senanges. En insistant sur les attitudes, le masque du fard, le port du vêtement, et en éliminant l'imprévu, il fige la personne dans un choix moral définitif, l'empêche à tout jamais de changer de cap et de se laisser prendre au piège de l'instant. Souvent, l'abstraction est poussée plus loin encore; l'auteur se borne aux caractéristiques morales du personnage, et le portrait se change en éthopée, comme dans cette présentation de Mme de Lursay:

Coquette jadis, même un peu galante, une aventure d'éclat, et qui avait terni sa réputation, l'avait dégoûtée des plaisirs bruyants du grand monde. Aussi sensible mais plus prudente, elle avait compris enfin que les femmes se perdent moins par leurs faiblesses que par le peu de ménagement qu'elles ont pour elles-mêmes; et que, pour être ignorés, les transports d'un amant n'en sont ni moins réels, ni moins doux. Malgré l'air prude qu'elle avait pris, on s'obstinait toujours à la soupçonner; et j'étais peut-être le seul à qui elle en eût imposé. (p. 53)

Le goût de la maxime et du portrait dans Les Egarements du coeur et de l'esprit rappelle que l'habitude des dissertations morales et de l'analyse psychologique est née dans les salons précieux et exprime le lien entre la littérature et les pratiques mondaines. Mais le maniement des figures n'offre pas seulement un intérêt sociologique. Il renvoie à une structure argumentative qui conditionne l'existence même du texte. Ch. Perelman a dénoncé à plusieurs reprises la réduction de la rhétorique à un catalogue de figures. Il souligne, dans un de ses plus récents ouvrages, que les anciens la concevaient "comme la technique par excellence, celle d'agir sur les autres hommes au moyen du logos, terme désignant d'une façon équivoque à la fois, la parole et la raison"; avec Gérard Genette, il juge significative l'absence d'une théorie des figures dans la Rhétorique d'Aristote. ${ }^{7}$ Raison et parole fusionnent en effet dans le dialogue, qui est un logos, ordre émergeant de la séparation, de l'affrontement de deux interlocuteurs ou de l'orateur et de son public, du même coup les réunissant (dia-légein). Comme l'a constaté Horst Wagner, le dialogue occupe une place plus importante dans Les Egarements que dans n'importe quel autre roman ou récit de Crébillon, et il représente plus de la moitié du texte. ${ }^{8}$ Pour Meilcour comme pour les autres personnages, aimer et parler sont presque la même chose; le discours de la séduction se substitue à l'amour-sentiment. Dans la première partie du roman, la possibilité, pour Meilcour, d'avouer son amour à Mme de Lursay, équivaut quasiment à la victoire. "Mais enfin que me demandez-vous?" interroge-t-elle. "Que vous croyiez que je vous aime ..., que vous me permettiez de vous le dire, et d'espérer qu'un jour je vous y verrai plus 
sensible." Et le mouvement de pudeur de Mme de Lursay se traduit, comme de juste, par le refus de parler: "Mais non, je n'ai plus rien à vous dire: je vous défends même de me deviner" (p. 90). Faisant allusion à ses tentatives auprès de Madame de . . ., et que Mme de Lursay a finalement fait échouer, Versac explique: "j'étais écouté convenablement, enfin: je persuadais" (p. 135). Mais c'est surtout dans la troisième partie que la persuasion coïncide avec la reddition, corps et âme, de l'objet convoité. Convaincu d'avoir affaire à une femme facile et d'être en droit de la mépriser, Meilcour presse Mme de Lursay de lui accorder un tête à tête; son discours devenu équivoque suggère que parler signifie faire des avances: "j'ai peut-être assez de choses à vous dire," prétend le jeune homme, "pour vous faire passer sans ennui le temps que je vous supplie de vouloir bien m'accorder" (p. 273). C'est effectivement ce soirlà que Mme de Lursay acceptera de se donner à lui. ${ }^{9}$

La conversation ne joue ce rôle déterminant dans l'univers du roman que parce que l'amour y est oblitéré par le lien social. Or les rapports sociaux apparaissent d'abord comme des rapports de domination. Aimer compte moins que séduire. Le tout, dans le monde, est de s'imposer, d'oser prendre même ce qui revient à autrui. Qu'importe si l'on ne peut rendre ce que l'on a reçu? L'homme du monde n'est point embarrassé d'acquitter ses dettes: il lui suffit de payer de mots. Les conseils de Versac à Meilcour ont le mérite d'être simples. Il faut, dit-il, se faire valoir. Comment? En affichant son mérite. "Surtout, parlons toujours, et en bien, de nous-mêmes: ne craignons point de dire et de répéter que nous avons un mérite supérieur." Risquons-nous de dégoûter à force de louer nos qualités? L'inconvénient compte peu, au regard des avantages: "Tout homme qui vous blâme de trop parler de vous, ne le fait que parce que vous ne lui laissez pas toujours le temps de parler de lui: plus modeste, vous serez martyr de sa vanité." Au demeurant, "il est plus sûr de subjuguer les autres, que de leur immoler sans cesse les intérêts de notre amour-propre" (p. 247).

Point n'est besoin d'être pénétré de son mérite pour en convaincre les autres. On peut même sans dommage se prendre intérieurement pour un imbécile, du moment que l'on paraît croire le contraire. Tout est dans ce qu'on dit, non dans ce qu'on sent: "en général vous ne pouvez assez vous emparer de la conversation." A-t-on l'esprit vide, cela ne doit pas encore empêcher de discourir, fût-ce sur les sujets qu'on ignore: "L'arrangement, ou plutôt l'abus des mots, tient lieu de pensées" (p. 251).

Prendre la parole revient ainsi à prendre le pouvoir. Parmi les préceptes que formule Varsac, il en est deux que Meilcour commence par juger contradictoires, mais qui dénotent une connaissance profonde des conditions dans lesquelles s'instaure l'“empire rhétorique". Le premier est d'imiter ce qu'on voit, d'adopter, même de flatter les préjugés d'autrui, c'est-à dire d'être rigoureusement conformiste. Le second, de se singulariser par des impertinences (pp. 243-244). Versac dévoile ici la face la plus cachée de son art. Il comprend admirablement que le rhéteur doit partir des lieux communs et en général s'y soumettre, tout en sachant en temps opportun les bafouer, puisqu'il lui convient autant d'attirer l'attention sur sa personne que d'être approuvé de ceux qui l'écoutent. 
Recettes valables non seulement pour la conversation, mais aussi pour les affaires de coeur. Car prendre le pouvoir, c'est d'abord s'emparer des femmes. C'est par elles qu'on parvient aux sommets, elles qu'on doit ménager pour avoir la faveur de l'opinion. Tel que Versac le décrit, l'amour peut être ou ne pas être un sentiment authentique. Dans tous les cas, l'homme du monde le transforme en exercice de manipulation:

Le moyen le plus simple et en même temps le plus agréable (de rendre son nom célèbre), est de paraître n'avoir dans tout ce qu'on fait que les femmes en vue, de croire qu'il n'y a d'agrément que ce qui les séduit, et que le genre d'esprit qui leur plaît, quel qu'il soit, est en effet le seul qui doive plaire. Ce n'est qu'en paraissant soumis à tout ce qu'elles veulent, qu'on parvient à les dominer. (p. 244)

L'amour, comme la conversation, est affrontement, compétition, parade, défi. C'est un discours à travers lequel chacun cherche à se mesurer, à jauger les forces de l'autre pour finalement le subjuguer. Le langage de la séduction usera d'un vocabulaire belliqueux, puisque l'amour tient de la stratégie: attaque, défense, conquête, résistance, faiblesse, combat, victoire, défaite, honneur, gloire, régner, succomber, soumettre, céder, sont des termes qui reviennent presque à chaque page du roman. Les hommes n'en ont d'ailleurs pas le privilège exclusif. Les femmes usent et abusent, tout aussi bien, des mots qui se rapportent à la guerre. Pour elles, les règles à suivre sont différentes. Si elles se gardent de prendre l'initiative, en se contentant de favoriser l'occasion qui donne envie de les attaquer, ce sont elles qui choisissent les vainqueurs. L'homme croit les soumettre, quand il ne fait que se prendre à leur piège. L'empire des femmes semble donc être la contrepartie de la domination des hommes. Elles dissimulent derrière le voile de la pudeur les manoeuvres qui assurent leur puissance. La rhétorique de la séduction est foncièrement trompeuse et elle cultive l'artifice pour faire oublier ses desseins véritables. Il s'agit, pour les femmes, de vaincre, tout en donnant l'impression d'être vaincues. Elles opposeront à l'homme de faux obstacles qui le persuaderont qu'on cède à son mérite alors que l'issue du combat était arrêtée d'avance. La vanité masculine trouve ainsi à se satisfaire, même si, en définitive, les femmes dictent les règles du jeu. "Plus elle m'oppose d'obstacles, plus ma gloire sera grande," avoue Meilcour à propos de Mme de Lursay (p. 74).

La vanité, qui mise tout sur le paraître, est le principal moteur des actions humaines, comme le langage est leur point d'appui. Les Egarements du coeur et de l'esprit illustrent à merveille la célèbre maxime de La Rochefoucauld: "il en est de tels qui n'auraient jamais été amoureux, s'ils n'avaient entendu parler d'amour." Dans la seconde partie du roman, Versac avertit Mme de Senanges que Mme de Lursay et Meilcour sont "bien ensemble" (p. 162), et il n'en faut pas davantage pour que la Senanges, toujours alléchée par une "conquête nouvelle" (p. 170), entreprenne de séduire Meilcour. Dans la troisième partie, Mme de Mongennes défend la réputation de Mme de Lursay, craignant que la Senanges, qui s'acharne à dénigrer sa rivale, ne parvienne à supplanter Mme de Lursay dans le coeur de Meilcour (pp. 213-218). Celui-ci, de son côté, a tout fait pour se débarrasser de Mme de 
Lursay depuis qu'il a rencontré Hortense; il a même refusé une invitation à la suivre à la campagne. Mais lorsque Mme de Lursay se résigne à ce changement de fortune, qu'elle adopte à son tour pour lui parler le ton de l'indifférence, Meilcour se sent piqué au vif, voire "malheureux" (p. 263), et il se remet à lui faire une cour effrénée.

Les échanges verbaux entre les personnages, conversations galantes, médisances, persiflages, explorent une figure de rhétorique fondamentale: l'allusion. Au début du roman, Meilcour n'osant déclarer son amour à Mme de Lursay, celle-ci cherche à l'encourager et à lui fournir des prétextes de lui parler. Elle met la conversation sur une comédie alors à l'affiche, dont elle loue un passage contenant une déclaration d'amour. Meilcour convient que cette partie a été bien traitée, d'autant que lui-même trouve difficile "de dire qu'on aime." La difficulté, observe Mme de Lursay, existe surtout pour la femme: "Pensez-vous, dans quelque désordre qu'elle sentît son coeur, qu'il lui convînt de parler la première, de s'exposer par cette démarche à se rendre moins chère à vos yeux, et à être l'objet d'un refus?" (p. 59). Il est clair qu'en posant cette question, Mme de Lursay pense à elle-même: la phrase tend à mettre Meilcour dans le coup, par le procédé de l'association ("vos yeux").

Peu à peu, l'allusion se précise, le dialogue prend un tour plus personnel, même si aucun des deux interlocuteurs n'admet franchement qu'il expose sa propre situation:

- Eh! comptez-vous pour rien, Madame, repris-je, l'embarras de le dire, surtout pour moi qui sens que je le dirais mal?

- Les déclarations les plus élégantes ne sont pas toujours, répondit-elle, les mieux reçues. On s'amuse de l'esprit d'un amant, mais ce n'est pas lui qui persuade; son trouble, la difficulté qu'il trouve à s'exprimer, le désordre de ses discours, voilà ce qui le rend à craindre. (p. 60)

Comme Meilcour néanmoins persiste dans son point de vue, Mme de Lursay en conclut qu'il trouve les déclarations embarrassantes parce qu'il en a une à faire (p. 61). L'étape suivante du dialogue conduit les personnages à formuler leurs sentiments comme s'il s'agissait de pures suppositions:

- Mais, Madame, si c'était une personne telle que vous que j'aimasse, à quoi me servirait-il de le lui dire?

- A rien peut-être, répondit-elle en rougissant.

- Je n'ai donc pas de tort, repris-je, de m'opiniâtrer au silence.

- Peut-être aussi réussiriez-vous: une personne de mon caractère peut, continua-t-elle, devenir sensible, et même plus qu'une autre. (p. 63)

Tant et si bien que, renonçant aux expressions fictives, Meilcour a finalement le courage de se découvrir: "Oserais-je, Madame, vous dire que je ne suppose rien?" (p. 64)

La plupart des conversations relatées dans le roman s'inspirent de ce modèle. Dans la dispute entre Mme de Senanges et Mme de Mongennes à propos de Mme de Lursay, il apparaît vite que celle-ci n'est qu'un prétexte. Le portrait peu flatteur que la Senanges fait de Mme de Lursay atteint par ricochet Mme de Mongennes, qui à son tour, prétend apprécier Mme de 
Lursay parce qu'elle s'identifie à elle et la compare avantageusement à Mme de Senanges: "Je conviens," reconnaît-elle, "qu'elle n'est plus de la première jeunesse; mais combien ne voit-on pas de femmes, beaucoup moins jeunes qu'elle, inspirer encore des sentiments, ou du moins chercher à les faire naître?" (214)

L'allusion (comme l'emploi du "on", mis pour "je" dans la conversation précieuse) est une forme de mise en abyme. Ce procédé joue, à vrai dire, un rôle majeur chez Crébillon; une étude plus poussée montrerait peut-être qu'il génère la totalité de son univers romanesque. Quand Meilcour voit pour la première fois Hortense de Théville à l'Opéra, il la regarde, ainsi que les gens sur lesquels elle jette les yeux: "Les miens se portaient aussitôt sur l'objet qu'elle avait paru vouloir chercher . . . Sans pénétrer le motif que me faisait agir, je conduisais, j’interprétais ses regards . .." (p. 76). Meilcour ne parle pas à Hortense, car il ne la connaît pas, mais à un ami qui est avec lui, et il cherche à briller dans la conversation dans l'espoir d'être entendu de la jeune fille (p. 77). Enfin, lorsqu'arrive le marquis de Germeuil, il les regarde se regarder.

Meilcour rencontrera une seconde fois Mlle de Théville aux Tuileries, accompagnée d'une dame. Il écoutera leur conversation. Tout en causant avec Hortense, la dame la questionne sur l'inconnu qui l'avait regardée à l'Opéra (p. 97-98).

Plus tard, chez Mme de Théville, Hortense lit un roman où il est question d'un amant malheureux parce qu'il s'imagine -à tort- n'être pas aimé. Elle raconte à Meilcour cette histoire, qui est probablement la leur (p. 190-191).

Après le séjour à Versailles, Meilcour retrouve Mme de Lursay avec Mlle de Théville. Il remarque que Mme de Lursay le regarde avec ironie pendant que lui-même observe Hortense (pp. 226-227).

La mise en abyme renvoie à une réalité qui se dérobe. Dans Les Egarements, le sentiment se perd dans les jeux de miroirs dont les personnages se renvoient mutuellement les reflets, captés dans les mots ou dans les regards. On a parfois comparé Crébillon à Proust et effectivement, chez les Meilcour, les Versac, les Senanges, les Mongennes, l'amour est entièrement socialisé comme l'est aussi, jusqu'à un certain point, l'amour de Swann pour Odette. ${ }^{10}$ On est épris parce qu'on croit l'être ou qu'on veut l'être, ou parce que l'autre vous néglige. Les gestes et la parure ont, dans ce contexte, la même fonction que les mots; messages que les personnages déchiffrent à l'aide du code social dont ils ont tous une parfaite connaissance. Après que Meilcour lui a avoué son amour, Mme de Lursay soupire, rougit, tourne vers lui des yeux languissants, se cache derrière son éventail et se tait (p. 64); s'il savait mieux les usages, le jeune homme aurait tôt fait d'interpréter ces façons comme une réponse positive. Voulant séduire Hortense, Versac, qui "avait la jambe belle . . . la fit valoir. Il rit le plus souvent qu'il put, pour montrer ses dents . .." (p. 159). Quand il verra Meilcour à l'Etoile, il lui apprendra qu'il faut avoir, dans le monde, "une négligence dans le maintien, qui, chez les femmes, aille jusques à l'indécence, et passe, chez nous, ce qu'on appelle aisance et liberté; tons et manières affectés, soit dans la vivacité, soit dans la langueur; l'esprit frivole et méchant, un discours entortillé . . ." (p. 254). 
Mais l'éducation sentimentale de Meilcour passe par plusieurs étapes. Au moment où il écrit ses mémoires, il est devenu cynique: Serge Gaubert note l'emploi fréquent d'analepses qui éclairent l'évolution du narrateur par rapport au jeune Meilcour. ${ }^{11}$ A son entrée dans le monde, Meilcour s'empêtre dans ses sentiments; il a besoin d'aimer, mais il ignore comment satisfaire ses désirs; il est timide, et ne sait quel objet choisir; il décide d'entreprendre la marquise de Lursay, parce qu'elle est la femme qu'il voit le plus souvent. La marquise lui enseigne à distinguer le respect de la timidité, à ménager sa délicatesse, à accorder la passion aux convenances. Les révélations de Versac sur le passé de Mme de Lursay, les conseils qu'il prodigue sur la manière de se conduire dans le monde le convaincront que la marquise a joué les fausses prudes et qu'il peut l'avoir à la hussarde. Mais il importe de remarquer que Versac n'aura pas le dernier mot. Mme de Lursay prouvera à Meilcour qu'il a manqué de finesse et qu'il a agi comme un mufle. Certes, elle n'est pas telle qu'il l'avait imaginée dans son ignorance et sa candeur. Meilcour n'est pas le premier homme qu'elle ait aimé. Mais elle n'est pas non plus telle que Versac l'a dépeinte: sans principes et sans moeurs. La fin du roman montre que Meilcour a eu tort de douter de la sincérité de Mme de Lursay, de la profondeur de ses sentiments. Elle avait dit: "je hais l'artifice" (p. III), et somme toute, avec raison. Lorsque Meilcour, pour se justifier d'avoir manqué un rendez-vous, avait pris les airs et le ton à la mode, elle avait su les lui reprocher: "Voilà de grands termes . . . si je n'exigeais de vous que des mots, j'aurais lieu d'être contente. Mais vous n'êtes pas de bonne foi . . ." (p. 86). Si prompt que soit Meilcour à suivre les traces de Versac, Mme de Lursay ne sera pas la dupe de ses paroles: elle cédera, non aux manoeuvres du "stratège", mais à la force du sentiment.

Crébillon a sévèrement dénoncé la rhétorique de Versac. "Il s'était fait," dit Meilcour, "un jargon extraordinaire qui, tout apprêté qu'il était, avait cependant l'air naturel" (p. 130). Mme de Lursay le condamne avec encore plus de véhémence: "Il parle," selon elle, "un jargon qui éblouit: il ne se connaît à rien, et juge de tout" (p. 142). Quant à Mme de Senanges, son alter ego, son aspect "ne présente plus aux yeux qu'un tableau de corruption qu'on ne peut regarder sans horreur"; comme Versac, elle utilise des "tournures de Cour, bizarres, négligées et nouvelles, ou renouvelées” (p. 152).

C'est à juste titre que Derrida dérive la mise en abyme de la métaphysique de la présence. Crébillon ne se borne pas à constater que l'amour se réduit à l'usage d'un jargon mis au service de la vanité, il le déplore et il réprouve les conduites dénaturées. Au fond, le roman tout entier justifie la distinction entre "plaire" et "toucher" (p. 54). A la mauvaise rhétorique dégagée de toute loi morale et de toute vérité, s'oppose une rhétorique soucieuse de respecter la raison et les sentiments authentiques. Le jeune Meilcour se leurrait sûrement en s'imaginant qu'il suffit d'être sincère pour faire naître l'amour. Mais la conduite de Mme de Lursay inflige un démenti à l'opinion de Versac: "Les femmes se rendent promptement, à peine attendent-elles qu'on les en prie" (p. 168).

A mi-chemin entre nature et culture, l'amour est à la fois un sentiment et un art. Il relève autant de l'instinct que de la sémiotique sociale. Présence, 
mais à renouveler par une attention constante, à empêcher de s'égarer dans les errements de l'esprit et les débordements du coeur.

\author{
Jean Terrasse \\ Université McGill
}

\title{
Notes
}

1 Le Dilemme du roman au XVIII e siècle. Etude sur les rapports du roman et de la critique (1715-1761) (New Haven: Yale Univ. Press, Paris: P.U.F., 1963), p. 15.

${ }^{2}$ Le Genre romanesque en France depuis l'apparition de la "Nouvelle Héloîse" jusqu'aux approches de la Révolution (Bruxelles: Maurice Lamertin, 1922), p. 78.

3 Tzvetan TODOROV, Littérature et signification (Paris: Librairie Larousse, 1967), p. 102.

${ }^{4}$ Jacques DERRIDA, De la Grammatologie (Paris: Les Editions de Minuit), p. 41.

5 Les Egarements du coeur et de l'esprit (Paris: Folio. 1977), p. 41. Toutes nos citations du roman seront tirées de cette édition.

"Manifeste du surréalisme, Paris, coll. "Idées", (1967). p. 44.

7 Chaïm PERELMAN, L'Empire rhétorique. Rhétorique et argumentation (Paris; Librairie philosophique J. Vrin, 1977). pp. 10-11. Voir surtout Chaïm PERELMAN et L. OLBRECHTS-TYTECA, La Nouvelle rhétorique. Traité de l'argumentation (Paris: P.U.F., 1958).

${ }^{8}$ Crébillon fils. Die Erzählerische Struktur seines Werkes (Munich: Wilhelm Fink Verlag, $1972)$, p. 68.

9 “'Sans aucun doute, les confidences osées, les médisances cruelles qui s'échangent dans les salons aristocratiques servent à exciter l'imagination érotique des auditeurs émoustillés, de même qu'elles fournissent matière aux tête-à tête des couples à mousser leurs désirs sexuels" (Ernest STURM, Crébillon fils et le libertinage au dix-huitième siècle (Paris: Nizet, 1970), p. 73.

10 Cf. Michel GILOT, "Les doux aveux de Crébillon," dans: Les Paradoxes du romancier: les "Egarements" de Crébillon (Grenoble, Presses Univ. de Grenoble, 1975), p. 91.

1 "Synchronie et diachronie ou la naissance du narrateur," dans Les Paradoxes du romancier, p. 44 . 